\title{
A little knowledge is a dangerous thing. So is a lot
}

\author{
Elena Ashikhmina, MD, PhD
}

See related article on pages e79-81.

Abdelaziz and colleagues report a case of an acute pulmonary embolism by right atrial myxoma during transesophageal echocardiography (TEE). ${ }^{1}$ The team showed excellent coordination in recognizing the problem and taking the patient to the operating room in a timely manner with a subsequent good outcome.

Although the authors claim to be the first to describe this complication, at least 2 reports of right atrial mass embolism during TEE (whether it was a tumor or a clot) are available. ${ }^{2,3}$ Not all of those cases had a happy ending. ${ }^{3}$ However, one detail is similar in all of the reports: Patients undergoing TEE already had a known diagnosis of right atrial mass by transthoracic echocardiography.

What prompted the physicians to order an additional diagnostic study such as TEE? The answer seems obvious: To further define the mass and its attachment. Obtaining additional knowledge seems like a right thing to do, but although it may not be harmful it is not always helpful. It might be also costly. Here is Albert Einstein's take: "A little knowledge is a dangerous thing. So is a lot."

Too many diagnostic tests being ordered by physicians is not a new problem. In 1979 and article by Hardison ${ }^{4}$ outlined different reasons why physicians order more and more tests. The majority of these reasons have nothing to do with finding the better way to treat patients, but rather fulfilling some obligations, following a protocol, or simply being "complete."

TEE has been shown to be a safe procedure in nonoperative settings, with $0.2 \%$ to $0.5 \%$ morbidity and less than
$0.01 \%$ mortality. ${ }^{5}$ However, anything above $0 \%$ seems to be high price to pay if it does not contribute to a patient's treatment.

In addition, the individual anesthetic plan for the TEE in the setting of right atrial cardiac masses should be thoroughly outlined. Currently we do not have enough

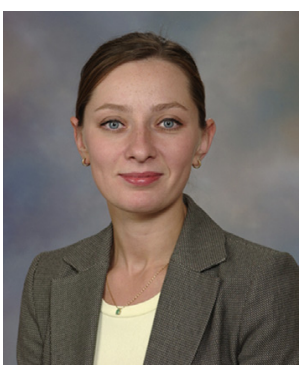
data to support risk and benefits of the topical anesthesia with conscious sedation versus general anesthesia in this patient population. This requires further investigation.

There are only few published reports of the right atrial mass embolization during TEE; therefore, its incidence could be underestimated and the mechanism is not fully defined. However, consequences could be devastating. The report by Abdelaziz and colleagues raises another red flag, emphasizing the need for a thoughtful approach when defining diagnostic plans for patients with intracardiac masses. ${ }^{1}$

\section{References}

1. Abdelaziz A, Abdelgawad A, Ramadan MM, Osama M. A new complication of transesophageal echocardiography: Pulmonary embolization of a right atrial myxoma. J Thorac Cardiovasc Surg. 2015;149:e79-81.

2. Kwak KD, Mosher SF, Willis CL, Kimura BJ. Witnessed embolization of a right atrial mass during transesophageal echocardiography: implications regarding the safety of esophageal intubation. Chest. 1999;115:1462-4.

3. Cavero MA, Cristóbal C, González M, Gallego JC, Oteo JF, Artaza M. Fatal pulmonary embolization of a right atrial mass during transesophageal echocardiography. J Am Soc Echocardiogr. 1998;11:397-8.

4. Hardison JE. To be complete. N Engl J Med. 1979;300:193-4.

5. Hilberath JN, Oakes DA, Shernan SK, Bulwer BE, D'Ambra MN, Eltzschig HK Safety of transesophageal echocardiography. J Am Soc Echocardiogr. 2010;23 1115-27.

From the Division of Pediatric Cardiac Anesthesia, Department of Anesthesiology, Perioperative, and Pain Medicine, Boston Children's Hospital, Boston, Mass.

Disclosures: Author has nothing to disclose with regard to commercial support.

Received for publication Jan 18, 2015; accepted for publication Jan 20, 2015; available ahead of print Feb 24, 2015.

Address for reprints: Elena Ashikhmina, MD, PhD, Division of Pediatric Cardiac Anesthesia, Department of Anesthesiology, Perioperative, and Pain Medicine, Boston Children's Hospital, 300 Longwood Ave, Boston, MA 02115 (E-mail: elena. ashikhmina@childrens.harvard.edu).

J Thorac Cardiovasc Surg 2015;149:e82

$0022-5223 / \$ 36.00$

Copyright (c) 2015 by The American Association for Thoracic Surgery

http://dx.doi.org/10.1016/j.jtcvs.2015.01.033 